\title{
NEUTRINOS FROM ICARUS
}

\section{Christian FARnese*, FOR The ICARUS Collaboration}

\author{
Dipartimento di Fisica e INFN, Università di Padova, Via Marzolo 8, I-35131,Padova, Italy \\ * corresponding author: christian.farnese@pd.infn.it
}

\begin{abstract}
Liquid Argon Time Projection Chambers are very promising detectors for neutrino and astroparticle physics due to their high granularity, good energy resolution and 3D imaging, allowing for a precise event reconstruction. ICARUS T600 is the largest liquid Argon (LAr) TPC detector ever built ( $\sim 600$ ton LAr mass) and is presently operating underground at the LNGS laboratory. This detector, internationally considered as the milestone towards the realization of the next generation of massive detectors ( $\sim$ tens of ktons) for neutrino and rare event physics, has been smoothly running since summer 2010, collecting data with the CNGS beam and with cosmics. The status of this detector will be shortly described together with the intent to adopt the LAr TPC technology at CERN as a possible solution to the sterile neutrino puzzle.
\end{abstract}

KEYWORDS: liquid argon, TPC, neutrino, CNGS, CERN, LNGS, sterile neutrino.

\section{The ICARUS T600 DETECTOR}

\subsection{Detection technique}

The Liquid Argon Time Projection Chamber (LAr-TPC), first proposed by C. Rubbia in 1977 [1], is a powerful detection technique that can provide a $3 \mathrm{D}$ imaging of any ionizing event. In a Liquid Argon TPC the ionization electrons produced by each ionizing event taking place in highly purified LAr can be transported by an uniform electric field and can be collected by 3 parallel wire planes, placed at the end of the drift path with wires oriented at different directions. The passage of charged particles through the LAr generates also a copious prompt ultra-violet scintillation light signal that can be detected using immersed PMTs. The LAr-TPC is a continuously sensitive and self triggering detector, characterized by high granularity and spatial resolution, similar to bubble chambers. The signal collected on the wire planes can provide a precise tridimensional reconstruction of the recorded particle trajectory. Moreover the LAr-TPC detector is an excellent calorimeter and it can provide an efficient particle identification based on the density of the energy deposition at the end of the particle range.

\subsection{Detector LAyout}

The ICARUS T600 LAr-TPC detector, presently taking data in Hall B of the Gran Sasso National underground Laboratory (LNGS), is the largest liquid Argon TPC ever built: it consists of a large cryostat split into two identical, adjacent modules, with internal dimensions $3.6 \times 3.9 \times 19.9 \mathrm{~m}^{3}$ each. Both modules house two Time Projection Chambers made of three parallel wire planes, $3 \mathrm{~mm}$ apart, the first with horizontal wires and the other two at $\pm 60^{\circ}$ from the horizontal direction. The two TPCs are separated by a common cathode and the distance between the cathode and the wire planes, corresponding to the maximal drift length, is $1.5 \mathrm{~m}$ equivalent to $1 \mathrm{~ms}$ drift time for a nominal drift field of $500 \mathrm{~V} / \mathrm{cm}$. Finally in order to collect the scintillation light produced by the events, an array of 74 PMTs (20 in West module and 54 in the East module) has been located behind the wire planes 2. The PMT signals are used not only for triggering but also to determine the absolute time of the ionizing event.

\subsection{Electron Lifetime}

The ICARUS detector is equipped with both gas and liquid recirculation systems containing standard $H y$ drosorb/Oxysorb ${ }^{\mathrm{TM}}$ filters in order to trap and keep at an exceptionally low level the electro-negative impurities, especially oxygen and nitrogen. The electron lifetime is continuously and automatically measured in real time by the charge signal attenuation as a function of the drift time along through-going muon tracks. The free electron lifetime is constantly above $5 \mathrm{~ms}$ in both the modules (see Fig. 1) corresponding to the remarkable value of $0.06 \mathrm{ppb}_{2}$ equivalent impurity concentration, resulting in a $17 \%$ charge attenuation at the maximum $1.5 \mathrm{~m}$ drift distance.

\subsection{TRIGGER SYSTEM}

The main ICARUS T600 trigger system is based on the analog sum of the signals from PMTs in the same chamber, with a defined photo-electron discrimination threshold for each of TPC chambers (100 phe in the West cryostat and 200 phe in the East cryostat). In particular for CNGS neutrino events, using the "Early Warning Signal" sent from CERN to LNGS $80 \mathrm{~ms}$ before each proton extraction, a $60 \mu \mathrm{s}$ gate is open at the expected proton extraction time, and the trigger is generated when the PMT sum signal is present in at least one TPC chamber within the gate. Using this source 

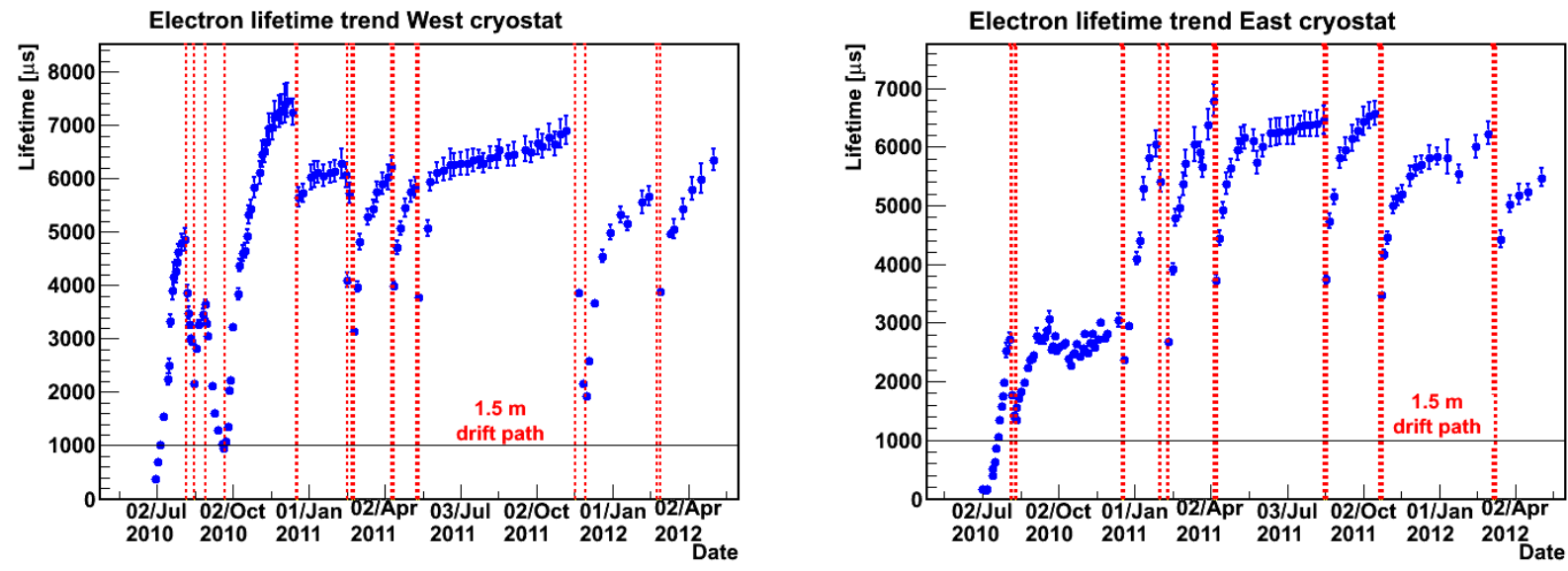

Figure 1. Free electron lifetime evolution with time, for both cryostats.
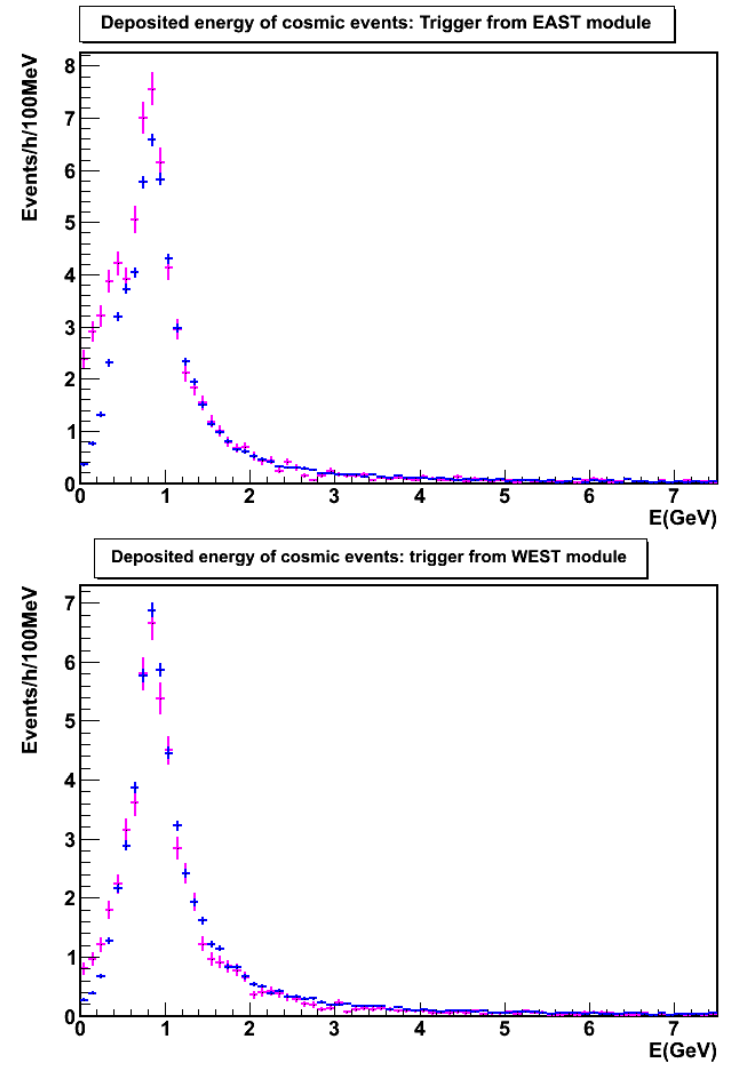

Figure 2. Comparison between the differential cosmic ray rates in the East (top) and West (bottom) modules, in blue the results for the events collected in July 2011 and in pink the results for events collected in March 2012, after the PMTs improvements carried out from December 2011 to March 2012.

of trigger about 80 events/day related to CNGS neutrino beam are recorded ( $\sim 1 \mathrm{mHz}$ rate). For cosmic ray events, the trigger is provided by the coincidence of the PMT sum signals of the two adjacent chambers in the same module. In Fig. 2, the differential c-ray rates in 2011 and 2012, as a function of deposited energy, are compared for both West and East module. Thanks to the recent improvement in the PMTs HV biasing scheme and signal treatment, an increase of the rates in the East half-module below about $1 \mathrm{GeV}$ has been obtained: an overall trigger rate of about $35 \mathrm{mHz}$ corresponding to 125 events/hour has been achieved on the full T600 (expected: 160 events/hour). A new FPGA called SuperDaedalus chip implementing a dedicated hit finding algorithm has been recently installed in order to improve the trigger efficiency both for CNGS and cosmic events at low energies (i.e. below $500 \mathrm{MeV}$ ). This new trigger system exploiting the local charge deposition on the TPC wires was used in 2012 run [4].

\subsection{Physics POTENTIALS AND ReSUlts FOR THE CNGS NEUTRINO RUNS}

LAr-TPCs are a very suitable detector for the study of rare events, such as neutrino oscillation physics and proton decay searches, because of the high spatial granularity (resolution of $\sim 1 \mathrm{~mm}^{3}$ on a overall active volume of $340 \mathrm{~m}^{3}$ for the ICARUS T600) and of the good calorimetric response $\left(\sigma_{E} / E \approx 11 \% / \sqrt{E(\mathrm{GeV})}\right)$. The ICARUS detection technique offers the possibility to detect "bubble chamber like" neutrino events produced by the CNGS neutrino beam from the CERN-SPS to Gran Sasso. ICARUS-CNGS run started in stable conditions on October 1st 2010, collecting $5.8 \times 10^{18}$ protons on target (p.o.t.) out of the $8 \times 10^{18}$ delivered by CERN up to November 22nd 2010. During the 2011 run an event statistics corresponding to $4.44 \times 10^{19}$ p.o.t. over the $4.78 \times 10^{19}$ p.o.t. delivered by CERN has been collected (Fig. 3), with detector live-time of $93 \%$ for CNGS exploitation: with this p.o.t. statistics $\sim 1280 \nu_{\mu} \mathrm{CC}$ and $\sim 400 \mathrm{NC}$ events are expected. An example of a $\nu_{\mu} \mathrm{CC}$ and of a $\nu_{\mu} \mathrm{NC}$ interaction collected during the 2011 run are shown in Figs. 5 and 6 .

Starting from the reconstruction of the muon produced in the CNGS $\nu_{\mu}$ interactions it is possible to verify the uniformity and stability of the detector: the muon tracks collected during a short period of the 2011 CNGS run have been reconstructed in 3D rejecting $\delta$-rays and showers along the track. The 


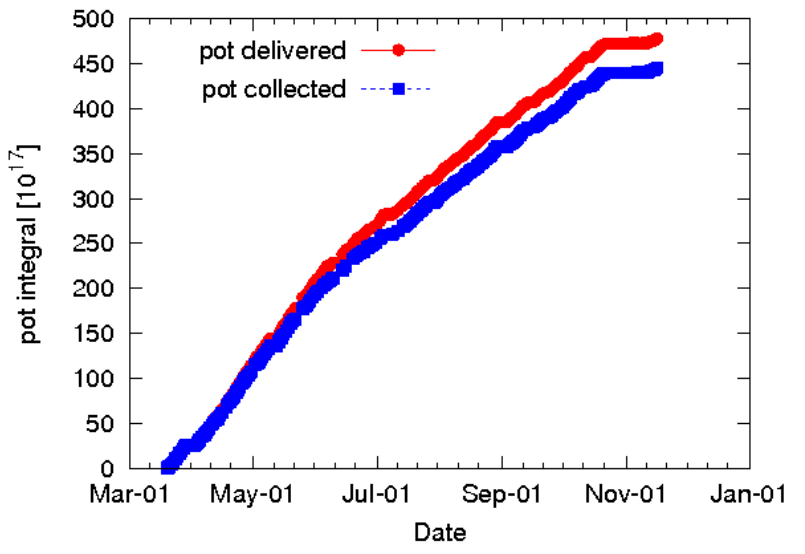

FiguRE 3. Integrated proton on target delivered to CNGS in the 2011 campaign (red). The corresponding integrated CNGS beam intensity (proton on target) that has been collected by the ICARUS T600 detector, taking into account the detector live-time, is also shown (blue).

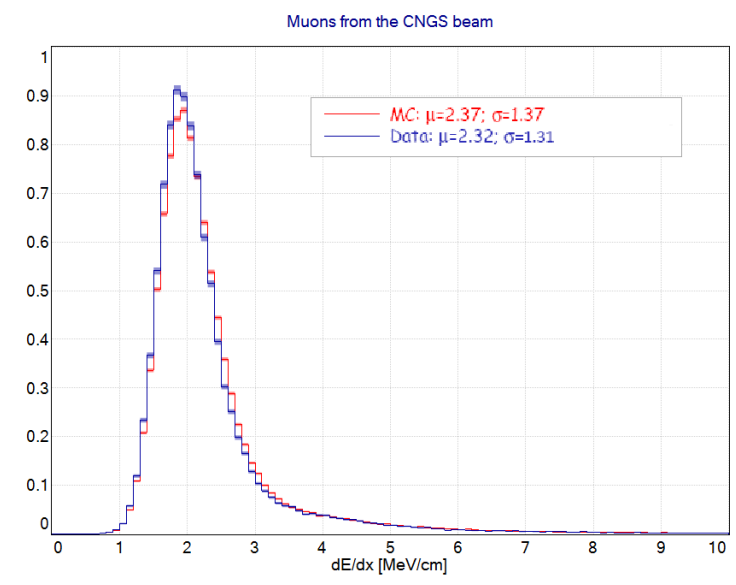

Figure 4. Comparison between the expected and the reconstructed energy deposition density distribution for muons in CNGS $\nu_{\mu} \mathrm{CC}$ interactions.

same analysis has been made also on MC events: the comparison between the $\mathrm{d} E / \mathrm{d} x$ distributions (Fig. 44) shows a very nice agreement both in shape and on average within $2 \div 3 \%$ demostrating a good detector calibration.

Thanks to the high resolution and granularity, the information redundancy and the particle identification capability, ICARUS can search for the $\nu_{\mu} \rightarrow \nu_{\tau}$ oscillation in the CNGS neutrino beam recognizing the $\tau$ decay on the basis of kinematical criteria. In addition, thanks to the capability to identify the interacting neutrino flavour and to discriminate the $\nu_{\mathrm{e}} \mathrm{CC}$ signal from the NC $\pi^{0}$ background with very high efficiency, ICARUS can also search for neutrino oscillation in LNSD parameter space.

ICARUS can also perform exclusive nucleon decay searches, in a background free mode thanks to its powerful background rejection: with an exposure of a few years ICARUS can improve the known limits in par- ticular for some "super-symmetric favored" nucleon decay channels 3]. Finally at least 100 CC atmospheric neutrino interaction per year are expected and can be fully reconstructed in ICARUS.

\section{SEARCH FOR SUPERLUMINAL NEUTRINO'S RADIATIVE PROCESSES IN ICARUS}

In september 2011 the OPERA Collaboration has reported an evidence of superluminal neutrino propagation (later disproved by the same collaboration): they observed that CNGS muon neutrinos arrive to Gran Sasso from CERN 60 ns earlier than expected for the travel at light speed. This result corresponds to $\delta \equiv\left(v_{v}^{2}-v_{c}^{2}\right) / v_{c}^{2} \approx 5 \times 10^{-5}[\underline{5}$.

Cohen and Glashow argued that if neutrinos are superluminal, they should loose their energy by producing photons and $\mathrm{e}^{+} \mathrm{e}^{-}$pairs, through $\mathrm{Z}^{0}$ mediated processes similar to the Cherenkov radiation [6]. To study in details the effects produced by the superluminal neutrino Cherenkov radiation, a full 3D simulation of the CNGS neutrino propagation from CERN to Gran Sasso has been performed using FLUKA for different values of the $\delta$ parameter and the expected rate for the $\mathrm{e}^{+} \mathrm{e}^{-}$pairs events have been estimated. Assuming the value of the parameter $\delta$ observed in the OPERA experiment, a strong deformation of the energy neutrino spectrum and in particular the full suppression of the neutrino events with an energy greater than $30 \mathrm{GeV}$ are expected. In addiction a very large amount of events containing $\mathrm{e}^{+} \mathrm{e}^{-}$pairs and photons should be found in the detector at the LNGS ( $\sim 10^{7}$ events $/ \mathrm{kt} / 10^{19}$ p.o.t. $)$. In order to verify the presence of these two effects using the ICARUS detector, the events corresponding to a total number of $4.9 \times 10^{18}$ p.o.t. for the 2010 run and of $1.04 \times 10^{19}$ p.o.t. for the 2011 run has been studied in detail by a visual scanning. The fiducial volume used for this analysis is 447 tons of Liquid Argon in 2010 and 434 tons in 2011. No events containing $\mathrm{e}^{+} \mathrm{e}^{-}$pairs has been observed in the ICARUS T600 detector as shown in Tab. 1. Furthermore the experimental energy deposition spectrum of neutrino events agrees with the MC expectation assuming $\delta=0$. The lack of the $\mathrm{e}^{+} \mathrm{e}^{-}$ pairs, combined with the no spectrum distorsion, allows to set the limit $\delta<2.5 \times 10^{-8}$ at $90 \%$ C.L. for multi-GeV neutrinos [7].

\section{Neutrino time of Flight MEASUREMENT WITH THE 2011 CNGS BUNCHED BEAM}

From October 21st to November 6th 2011 the CERN CNGS neutrino beam has been operated in a new, lower intensity mode that allows a very accurate neutrino time of flight measurement on an event by event basis. The bunched beam structure was characterized by 4 LHC-like extractions with a narrow width $(\sim 3 \mathrm{~ns}$ FWHM) separated by 524 ns, with $\sim 10^{12}$ p.o.t. per 


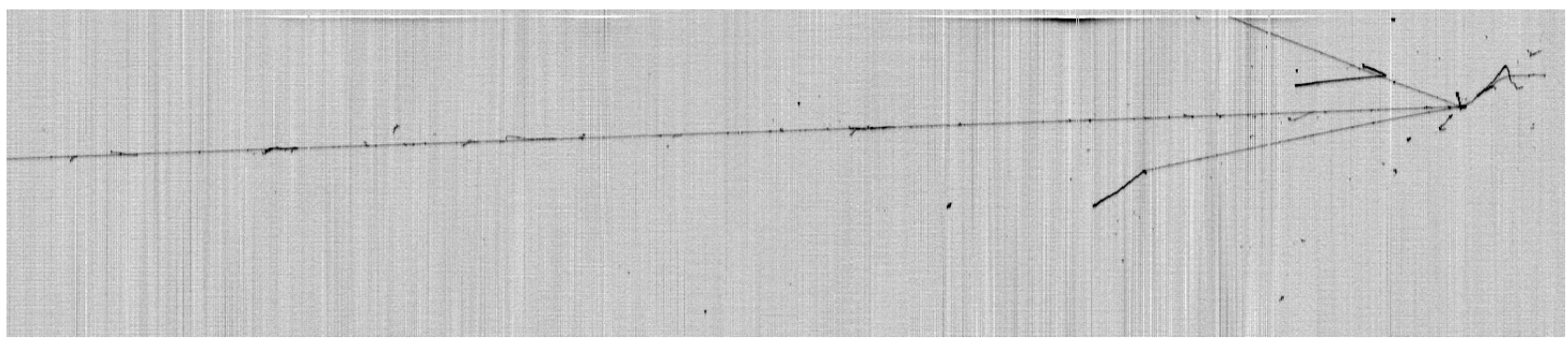

FiguRE 5. Example of a CC muon neutrino interaction collected during the 2011 run: the long muon track coming out from the primary vertex is clearly visible.

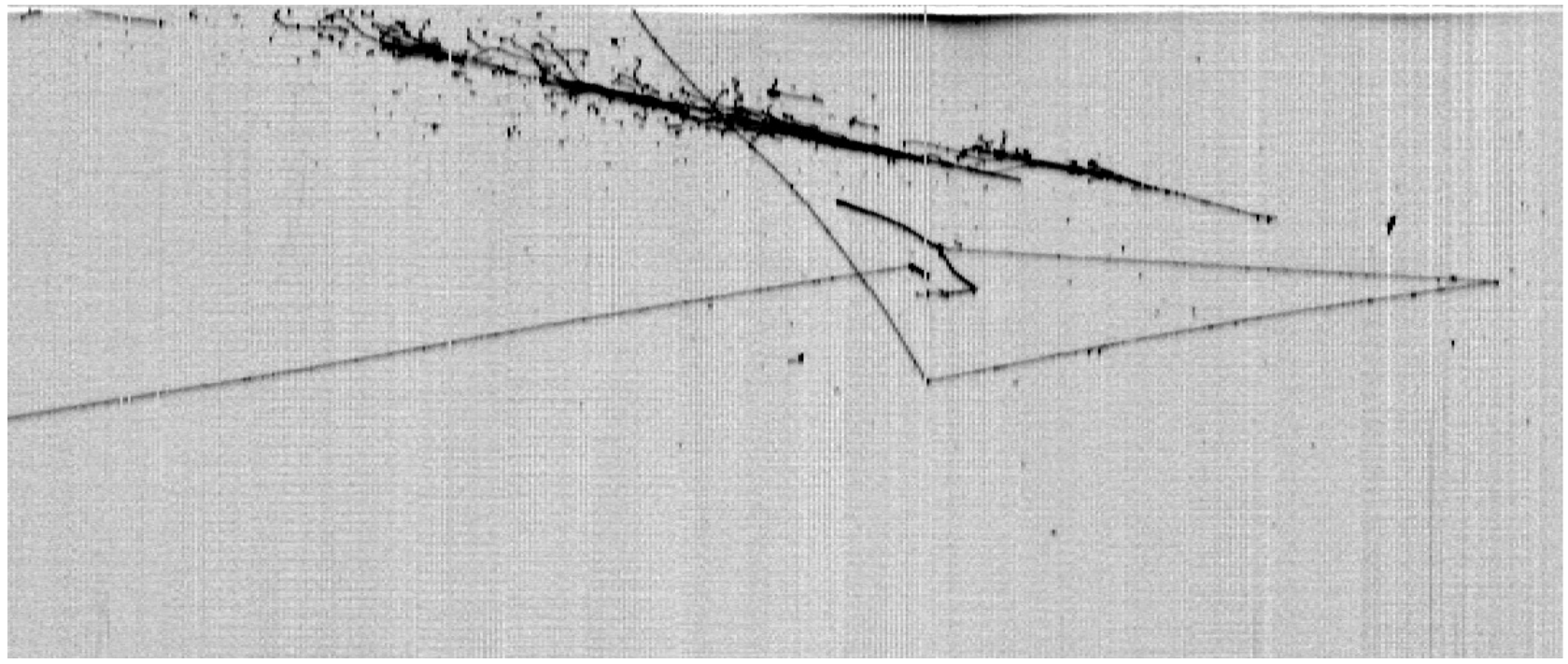

Figure 6. Example of a NC muon neutrino interaction collected during the 2011 run.

pulse. Because of a problem related to the "Early Warning signal", ICARUS started to collect neutrino data with this new beam from October 31st. 7 events has been collected: two $\nu_{\mu} \mathrm{CC}$ interaction and one $\mathrm{NC}$ event with the primary vertex contained in ICARUS fiducial volume and 4 through going muons generated by a neutrino interaction in the upstream rock. For each event, the neutrino time of flight tof ${ }_{\nu}$ has been calculated as the difference between the event time in the ICARUS detector $T_{\text {stop }}$ and the time $T_{\text {start }}$ of the proton transit at the Beam Current Transformer accounting for the additional time related to the nearest proton bunch. The use of the GPS-based timing system linking CERN to LNGS allows the time measurement with uncertainty of a few nanoseconds. In order to improve the precision of the neutrino arrival time, the ICARUS PMT system has been equipped with an additional PMT-DAQ system: at each CNGS trigger the PMT-Sum signals and the absolute time of the LNGS PPmS signal are stored allowing for the determination of the absolute neutrino arrival time in the detector within few ns precision. Moreover for each event the distance from the closest PMT and the position of the interaction vertex along the detector length has been evaluated and used to obtain a more precise arrival time estimation. Finally for each event the difference $\delta t$ between the expected time of flight considering the speed of light and the neutrino time of flight is calculated.

The average value of the parameter $\delta t$ obtained from the 7 events is $\delta t=+0.3 \mathrm{~ns} \pm 4.9 \mathrm{~ns}$ (stat.) $\pm 9 \mathrm{~ns}$ (syst), compatible with the Lorentz invariance $(\delta t \sim 0)[8]$.

\section{ICARUS AFTER CNGS2: A NEW APPROACH TO STERILE NEUTRINOS AT CERN/SPS}

The recent observations of an electron excess in a anti- $\nu_{\mu}$ beam, made by the LSND and MiniBooNE experiments, and of an apparent disappearance signal in the anti- $\nu_{\mathrm{e}}$ events collected by the reactor neutrino experiments, seem to suggest the presence of invisible "sterile" neutrinos, in addition to the three well known physical neutrino $\nu_{\mathrm{e}}, \nu_{\mu}$ and $\nu_{\tau}$. The ideal device to try to solve at the same time all these anomalies is the LAr-TPC thanks to the detection capability to recognize the genuine electron neutrino events combined with the high level of rejection of the associated background events due for example to $\mathrm{NC} \pi^{0}$ interactions. In addition the detector granularity and energy resolution provided by LAr TPCs are excellent for neutrino events at low energy (i.e. below $3 \mathrm{GeV}$ ). For these reasons a new experimental search, based on two strictly identical LAr-TPC detectors and two mag- 


\begin{tabular}{|l|l|l|l|}
\hline Rates & Observed & Expected $\boldsymbol{\delta}=0$ & Expected $\boldsymbol{\delta}=5 \times 10^{-5}$ \\
\hline $\mathbf{C C}$ & 308 & $315 \pm 5$ & $98.1 \pm 2$ \\
\hline $\mathbf{N C}$ & 89 & $93.1 \pm 3$ & $33 \pm 1$ \\
\hline$\nu_{\mathbf{\mu}} \mathbf{C C} E_{\text {dep }}>25 \mathbf{G e V}$ & 25 & $18 \pm 1.3$ & $<10^{-6}$ \\
\hline $\mathbf{e}^{+} \mathbf{e}^{-}$pairs & 0 & 0 & $7.4 \times 10^{6}$ \\
\hline
\end{tabular}

TABLE 1. Expected and observed neutrino and $\mathrm{e}^{+} \mathrm{e}^{-}$pairs rates for the ICARUS T600 detector [7].

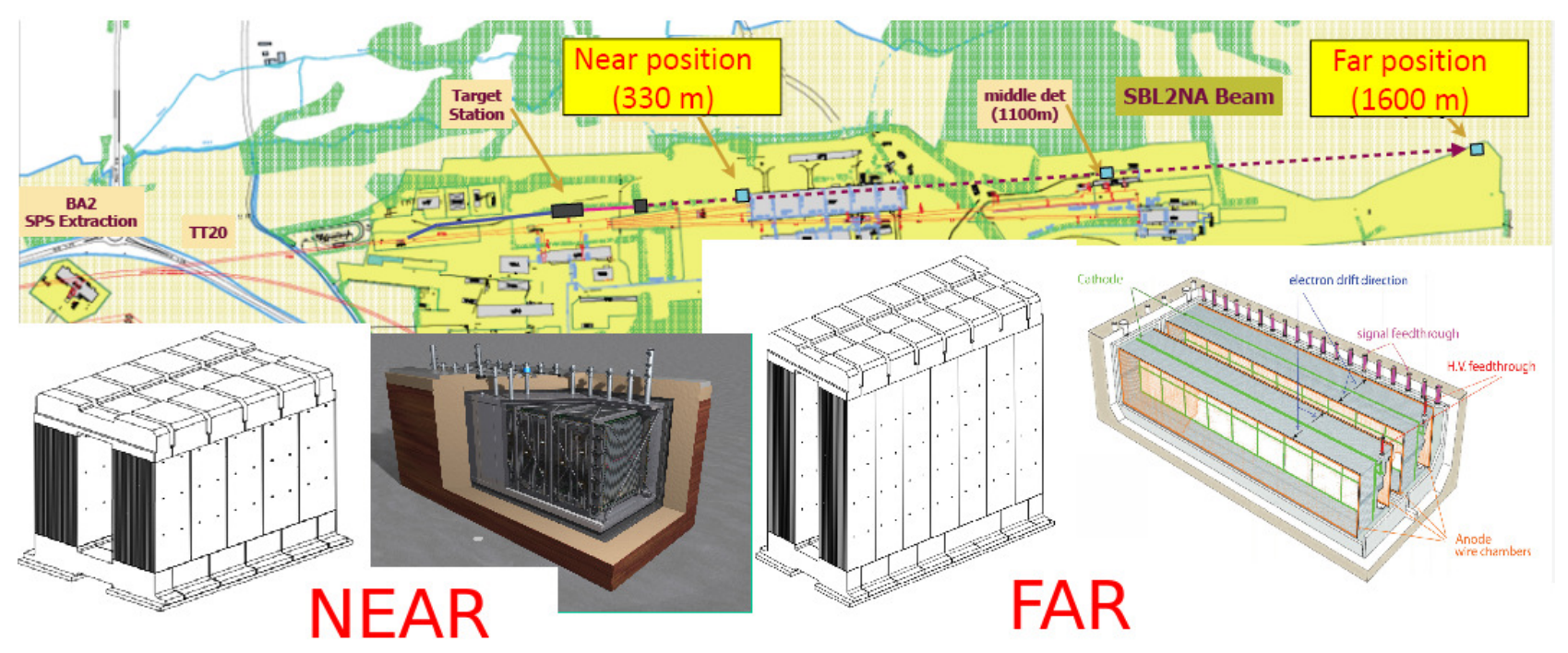

FIGURE 7. Scheme of the new neutrino facility proposed in the CERN north area 9].

netic spectrometers located at different distances on axis on a neutrino beam is proposed at CERN/SPS after the ICARUS T600 exploitation at the Gran Sasso Laboratories. A complete description of this new experiment can be found in the technical proposal [9]. A simple scheme of the neutrino facility located in the CERN north area is shown in Fig. 7] the ICARUS T600 detector will be moved from the Gran Sasso to CERN and located at $1600 \mathrm{~m}$ from the proton target (FAR position).

At the same time, a new LAr-TPC detector, identical to the ICARUS T600 but of smaller size (150t), will be built and located at $330 \mathrm{~m}$ (NEAR position) from the target. The two NESSiE spectrometers will be placed downstream of the LAr-TPCs in order to greatly complement the physics capabilities of this experiment. The neutrino beam will be produced by a $100 \mathrm{GeV}$ proton beam fast extracted from the SPS. The anti-neutrino beam can be produced simply inverting the current in the horn used to focus the beam. The resulting neutrino CC event energy will be centered around $2 \mathrm{GeV}$. In absence of oscillations the comparison between the neutrino spectra in the near and in the far position shows an excellent agreement for the $\nu_{\mathrm{e}}$ events and a fairly good agreement for the $\nu_{\mu}$ events, apart some small beam related spatial corrections. Therefore an exact observed proportionality between the two $\nu_{\mathrm{e}}$ spectra implies directly the absence of neutrino oscillations over the measured interval of $L / E$ without any need of Monte Carlo corrections: any difference between the Near and Far observed spectra would be a direct proof of neutrino oscillations and both the mixing angle and the mass difference can be determined separately. At the same time the determination of the muon charge by the spectrometers allows the full discrimination between the $\nu_{\mu}$ and $\bar{\nu}_{\mu}$ events. Thanks to the unique feature described and to the properties of the LAr-TPC and of the NESSiE magnetic spectrometers, the proposed experiment can provide a full exploration of the LSND allowed region and can also study the possible oscillatory disappearance both in the $\nu_{\mu}$ and the $\nu_{\mathrm{e}}$ initial signal studying both the neutrino and anti-neutrino beam [9].

\section{Conclusions}

The ICARUS-T600 detector installed underground at LNGS, is taking data with the CNGS neutrino beam since October 2010 in a stable condition searching both for $\nu_{\mu} \rightarrow \nu_{\tau}$ and the $\nu_{\mu} \rightarrow \nu_{e}$ oscillation as well as for atmospheric neutrinos and proton decay events. Its unique imaging capability, the spatial and calorimetric resolution and the capability to separate the shower produced from $\pi^{0}$ and from electrons allow to reconstruct and identify events in a new way with respect to the other current neutrino experiments. The successful start and data taking of the ICARUS-T600 detector surely represents a major milestone towards the realization of large mass LAr-TPC detectors for neutrino and astroparticle physics. 
Recently, the employment of the LAr-TPC technique, combined with the use of the magnetic spectrometers has been proposed at CERN-SPS after the ICARUS-T600 exploitation at LNGS in order to definitely solve the sterile neutrino puzzle.

\section{ACKNOWLEDGEMENTS}

I thank first of all the Organizing Committee for the possibility to present my talk during the VULCANO 2012 Workshop. I would like also to thank all the ICARUS Collaboration for the contributions in the preparation of the talk and of this proceeding.

\section{REFERENCES}

[1] C. Rubbia, The Liquid-Argon Time Projection Chamber: A new Concept for Neutrino Detector, CERN-EP/77-08 (1977)

[2] A. Ankowski et al. [ICARUS Collab.] Nucl. Instr. and Meth. A556 (2005) 146

[3] F. Arneodo et al.[ICARUS Collab.], ICARUS initial physics program, LNGS P28/01, LNGS-EXP 13/89 add. 1/01; Cloning of T600 modules to reach the design sensitive mass, LNGS-EXP 13/89 add. 2/01

[4] Baibussinov B. et al., A hardware implementation of Region-of-Interest selection in LAr-TPC for data reduction and triggering, JINST 5, P12006 (2010)

[5] T.Adam et al., [OPERA Collab.], arXiv:1109,4897v2, 2011

[6] A.G.Cohen, S.L. Glashow, Phys. Rev. Lett. 107(2011) 181803
[7] M. Antonello et al.[ICARUS Collab.], A search for the analogue to Cherenkov radiation by high energy neutrinos at superluminal speed in ICARUS, Physics Letters B 711(2012) 270-275

[8] M. Antonello et al.[ICARUS Collab.], Measurement of the neutrino velocity with the ICARUS detector at the CNGS beam, Physics Letters B 713(2012) 17-22

[9] M. Antonello et al.[ICARUS and NESSiE Collab.], Search for "anomalies" from neutrino and anti-neutrino oscillations at $\Delta \mathrm{m}^{2} \approx 1 \mathrm{eV}^{2}$ with muon spectrometers and large LAr-TPC imaging detectors,

CERN-SPSC-2012-010 and SPSC-P-347 (2012)

\section{Discussion}

Fernando Ferroni - Can you comment more on what the magnetic spectrometers bring to the measurement of sterile neutrinos?

Christian Farnese - Thanks to the use of magnetic spectrometers in the new proposed experiment at CERNSPS, the $\nu_{\mu}$ disappearance signal can be studyed in a better way. The determination of the muon charge with the spectrometers allows for the full discrimination of the $\nu_{\mu}$ from the $\bar{\nu}_{\mu}$ signal increasing the statistics and sensitivity for this physics item. Finally, combining the muon reconstruction based on the multiple scattering in the LAr-TPCs with the results from the spectrometer reconstruction a precise measurement for the muons can be obtained. 